\title{
Comportamento da Freqüência Cardíaca e da Sua Variabilidade Durante as Diferentes Fases do Exercício Físico Progressivo Máximo
}

\section{Denise de Oliveira Alonso, Claudlia Lucia de Moraes Foriaz, Liliane Onda Rezende, Ana Maria F. W. Braga, Antonio Carlos Pereira Barretto, Carlos Eduardo Negrão, Maria Urbana Pinto Brandão Rondon}

São Paulo, SP

\begin{abstract}
Objetivo - A variabilidade da freqüencia cardíaca (VFC) tem sido estudada em repouso, como meio nãoinvasivo para avaliação da regulação autonômica cardíaca, sendo que sua diminuição está relacionada a maior risco cardiovascular. Entretanto, durante o exercício, quando ocorrem importantes alterações neurais, seu comportamento deve ser melhor documentado. Estudamos o comportamento da freqüencia cardíaca $(F C)$ e da sua variabilidade durante as diferentes fases metabólicas do exercicio fisico progressivo máximo, em jovens.

Métodos - Dezessete homens (28土6 anos) realizaram teste ergoespirométrico máximo em cicloergômetro $(30 \mathrm{~W} /$ 3 min), determinando-se a FC e a VFC (desvio-padrão) através da onda eletrocardiográfica, amplificada e gravada batimento a batimento em computador, numa freqüência da $125 \mathrm{~Hz}$ (AT/Codas).

Resultados - $A$ FC aumentou concomitantemente ao aumento da intensidade do exercício. A VFC diminuiu progressivamente, atingindo niveis significantes em relação ao repouso a partir de $60 \%$ do consumo de oxigênio do pico do exercício, a partir de 45-60\% da potência máxima e a partir da intensidade do limiar anaeróbio, estabilizando-se nos periodos subseqüentes.

Conclusão-Nossos resultados sugerem que a VFC medida pelo desvio-padrão da FC diminui em fases do exercício nas quais o aumento da FCédeterminado, principalmente, por retirada vagal.
\end{abstract}

Palavras-chave: frequiência cardíaca, variabilidade da freqüência cardíaca, exercício

\section{Heart Rate Response and Its Variability During Different Phases of Maximal Graded Exercise}

Purpose - Heart rate variability (HRV) has been studied at rest as a non-invasive tool for the assessment of cardiac autonomic control and, its attenuation is related to cardiovascular risk. However, during exercise, when important neural changes take place, HRV behaviour is not well established. The aim of this investigation was to study the heart rate (HR) and HRV responses during the different metabolic phases of maximal graded exercise in young men.

Methods - Seventeen men (age $28 \pm 6$ years) were submitted to a graded cardiopulmonary exercise test in a cycloergometer (30W/3min). To study HR and HRV (standard-deviation), the eletrocardiographic signal was amplified and acquired beat-to-beat in a computer at a frequency of $125 \mathrm{~Hz}$ (AT/Codas).

Results - The HR increases concomitantly to the increase in exercise intensity. The HRV was significantly lower than rest values after exercise intensities of $60 \%$ of peak exercise oxygen uptake, $45-60 \%$ of maximal power and, after anaerobic threshold intensity.

Conclusion - This results suggest that HRV measured by standard deviation of HR decreases during exercise phases when HR increment is determined mainly due to vagal withdrawal.

Key-words: heart rate, heart rate variability, exercise

Arq Bras Cardiol, volume $71\left(n^{0}\right.$ 6), 787-792, 1998

A variabilidade da freqüência cardíaca (VFC) tem sido utilizada como meio não-invasivo de avaliação do controle

Instituto do Coração do Hospital das Clínicas - FMUSP

Correspondência: Denise de Oliveira Alonso - Incor - Av. Dr. Enéas C. Aguiar, 44 - 05403-000 - São Paulo, SP

Recebido para publicação em 27/4/98

Aceito em 14/10/98 neural do coração ${ }^{1-4}$. Estudos recentes ${ }^{5-8}$ têm demonstrado que a diminuição da VFC está relacionada a um maior índice de morbidade e mortalidade cardiovascular. Por essas razões, muitos autores têm se ocupado em utilizar manobras respiratórias ${ }^{9}$, mudanças de posição ${ }^{10,11}$ e bloqueios farmacológicos do sistemas nervosos simpático e parassimpático ${ }^{10}$, na tentativa de investigar a VFC. 
Oexercício físico, em si, é um comportamento que provoca importantes modificações no funcionamento do sistema cardiovascular e em seus mecanismos de ajustes autonômicos ${ }^{12-15}$. Assim, o estudo da VFC durante o exercício físico agudo pode permitir uma análise adicional e nãoinvasiva do controle neural da freqüência cardíaca $(\mathrm{FC}) \mathrm{du}-$ rante esse comportamento.

Estudos recentes têm demonstrado que o exercício físico progressivo, em indivíduos sedentários ${ }^{16-18}$, treinados ${ }^{19-21}$ e cardiopatas ${ }^{22,23}$ provoca uma diminuição no sistema nervoso parassimpático e um aumento no sistema nervoso simpático, que controlam a FC. Entretanto, em muitos desses estudos ${ }^{16,17,20,21}$, empregou-se um protocolo de incremento contínuo de potência (protocolo de rampa), o que não permite uma estabilização da FC em cada estágio do exercício - condição necessária para uma avaliação adequada da $\mathrm{VFC}^{24}$. Além disso, o estudo do comportamento da VFC, relacionando-o às diferentes intensidades e fases metabólicas do exercício físico progressivo, como a intensificação do metabolismo anaeróbio [limiar anaeróbio (LA)] e a descompensação da acidose metabólica [ponto de compensação respiratória (PCR)] ainda precisa ser melhor documentado.

Desta forma, o objetivo desta investigação foi analisar o comportamento da FC e da sua variabilidade durante as diferentes fases metabólicas do exercício físico progressivo máximo em indivíduos jovens saudáveis.

\section{Métodos}

A amostra foi constituída por 17 indivíduos jovens, saudáveis, sedentários, do sexo masculino, com idade média de $28 \pm 6$ anos, peso de $81 \pm 8 \mathrm{~kg}$, estatura de $182 \pm 5 \mathrm{~cm}$ e índice de massa corporal de $24 \pm 2 \mathrm{~kg} / \mathrm{m}^{2}$. Todos os indivíduos foram previamente informados sobre os procedimentos experimentais e concordaram na sua participação.

Assim que chegaram ao laboratório, os indivíduos realizaram um eletrocardiograma de repouso, com registro das 12 derivações padrão. A seguir, foram posicionados no cicloergômetro (Mijnhardt, Ken III/Medfit, modelo MFC400L) e orientados a realizar as manobras respiratórias (apnéia inspiratória, apnéia expiratória e hiperpnéia), antes de iniciarem o protocolo de exercício máximo.

$\mathrm{O}$ teste de esforço (TE) foi precedido por $3 \mathrm{~min}$ de repouso com o indivíduo sentado no cicloergômetro. Em seguida, iniciou-se o exercício, utilizando-se um protocolo escalonado, com incrementos de 30 Watts a cada $3 \mathrm{~min}$ até a exaustão. Prosseguiu-se um período de $6 \mathrm{~min}$ de recuperação, sendo que o $1^{\circ}$ minuto foi realizado com $50 \%$ da potência máxima alcançada, o $2^{\circ}, \operatorname{com} 25 \%$ da potência máxima e o $3^{\circ}$, com potência livre. Do $4^{\circ}$ ao $6^{\circ}$ minuto de recuperação o indivíduo permaneceu sentado em repouso no cicloergômetro.

Simultaneamente ao TE máximo, foram continuamente coletados o fluxo de ar expirado e amostras dos gases inspirados e expirados pelo indivíduo a cada ciclo respiratório, através de um analisador de gases computadorizado $(2001 \mathrm{Cad} /$ Net System - Medical Graphics Corporation - MGC). A partir destes dados, foram calculados a ventilação pulmonar
(VE), o consumo de oxigênio $\left(\mathrm{VO}_{2}\right)$, a produção de dióxido de carbono $\left(\mathrm{VCO}_{2}\right)$, a pressão parcial final de oxigênio $\left(\mathrm{PetO}_{2}\right)$, a pressão parcial final de dióxido de carbono $\left(\mathrm{PetCO}_{2}\right)$, a razão de troca respiratória (RER - $\mathrm{VCO}_{2} / \mathrm{VO}_{2}$ ), o equivalente ventilatório de oxigênio $\left(\mathrm{VE} / \mathrm{VO}_{2}\right)$ e o equivalente ventilatório de dióxido de carbono $\left(\mathrm{VE} / \mathrm{VCO}_{2}\right)$. Estes dados foram agrupados em médias de 60s, do repouso ao pico do exercício.

O consumo máximo de oxigênio foi definido como o $\mathrm{VO}_{2}$ obtido no pico do exercício $\left(\mathrm{VO}_{2}\right.$ pico), quando o indivíduo se encontrava em exaustão.

OLA foi determinado no minuto durante o exercício em que as curvas de $\mathrm{Pet} \mathrm{O}_{2} \mathrm{eVE} / \mathrm{VO}_{2}$ atingiram o seu valor mínimo antes de começarem a subire o RER apresentou um incremento não-linear ${ }^{25}$.

OPCR foi determinado no minuto durante o exercício em que a curva de VE/ $\mathrm{VCO}_{2}$ atingiu o seu valor mínimo, antes de começar a subir, e a de $\mathrm{PetCO}_{2}$ atingiu o seu maior valor antes de começar a diminuir ${ }^{25}$.

O sinal eletrocardiográfico de FC foi continuamente monitorizado, através das derivações MC5, D2 modificadae V2 modificada, num eletrocardiógrafo (Tecnologia Eletrônica Brasileira - TEB, modelos SM 310 e SM 400). Durante todo o período experimental, o sinal eletrocardiográfico da derivação MC5 foi pré-amplificado (General Purpose Amplifier/Stemtech, Inc, GPA-4, modelo 2), em seguida convertido de analógico para digital através de um conversor (Stemtech, Inc.), gravado batimento a batimento em um computador (Gateway 2000 4DX2-50V), e analisado numa frequiência de $125 \mathrm{~Hz}$, através do programa AT/Codas. Este programa permite o registro da FC batimento a batimento e sua posterior análise através do software excel. Batimentos fora do ritmo sinusal foram visualmente identificados por um técnico e excluídos da análise.

Os intervalos R-R do sinal eletrocardiográfico registrado batimento a batimento foram agrupados em intervalos de $1 \mathrm{~min}$, do repouso até o pico do exercício. Em cada um destes intervalos de $1 \mathrm{~min}$ foram calculados a média dos intervalos R-R - que correspondeu ao valor da FC de cada minuto - e o desvio-padrão dos mesmos intervalos $\mathrm{R}-\mathrm{R}$ - considerado como a VFC de cada minuto.

Os resultados obtidos de FC e de VFC foram analisados em relação ao impacto metabólico do exercício progressivo, em relação ao aumento relativo do $\mathrm{VO}_{2}$ e em relação ao aumento relativo da potência empregada no cicloergômetro durante o exercício.

Para se estudar a influência do impacto metabólico do exercício progressivo, os comportamentos da FC e da VFC foram analisados em repouso (no $2^{\circ}$ minuto de coleta de dados, antes do início do TE), no minuto em que foi determinado o LA, no minuto em que foi determinado o PCR e no minuto final de exercício.

Os valores de $\mathrm{VO}_{2}$ obtidos em médias de 60 s foram utilizados para o cálculo da equação da reta de regressão linear entre os valores de $\mathrm{VO}_{2}$ e o minuto de exercício correspondente. Através da equação da reta de regressão linear foi calculado o valor de $\mathrm{VO}_{2}$ correspondente ao minuto final de exercício ( $\mathrm{VO}_{2}$ fin). A partir deste valor, foram calculados os valores de 
$\mathrm{VO}_{2}$ relativos, correspondentes aos intervalos de 10, 20, 30, $40,50,60,70,80,90$ e $100 \%$ do $\mathrm{VO}_{2}$ fin. Para cada valor de $\mathrm{VO}_{2}$ relativo obtido, verificaram-se os valores de FC e de VFC, ocorridos no minuto do TE, em que o valor de $\mathrm{VO}_{2}$ ocorreu.

A partir do valor de potência máxima (Wmax) atingida por cada indivíduo no TE, foram calculados intervalos relativos de 20 a $35 \%$, de 45 a $60 \%$ e de 70 a $85 \%$ da Wmax. Estes intervalos foram escolhidos de forma que todos os indivíduos apresentassem somente um estágio de potência do protocolo de teste dentro de cada intervalo. Para análise da FC e da VFC foram utilizados os valores referentes ao $3^{\circ} \mathrm{mi}-$ nuto de cada estágio.

Para análise descritiva dos dados foram utilizados média \pm desvio-padrão. Na análise do comportamento da FCe VFC foi utilizada a análise de variância de um caminho para medidas repetidas, tendo como fator principal o estágio de exercício, com nível de significância aceito de $\mathrm{P}<0,05$. Quando encontrada diferença significante, foi realizado o teste post-hoc de Scheffè, para identificação das diferenças.

\section{Resultados}

O grupo atingiu uma capacidade física máxima de $34,1 \pm 5,7 \mathrm{mlO}_{2} \mathrm{~kg}^{-1} \mathrm{~min}^{-1}$ e a Wmax no TE foi de $199 \pm 30 \mathrm{~W}$.O LA foi atingido em $45 \pm 7 \%$ do $\mathrm{VO}_{2}$ pico e em $66 \pm 7 \%$ da FC

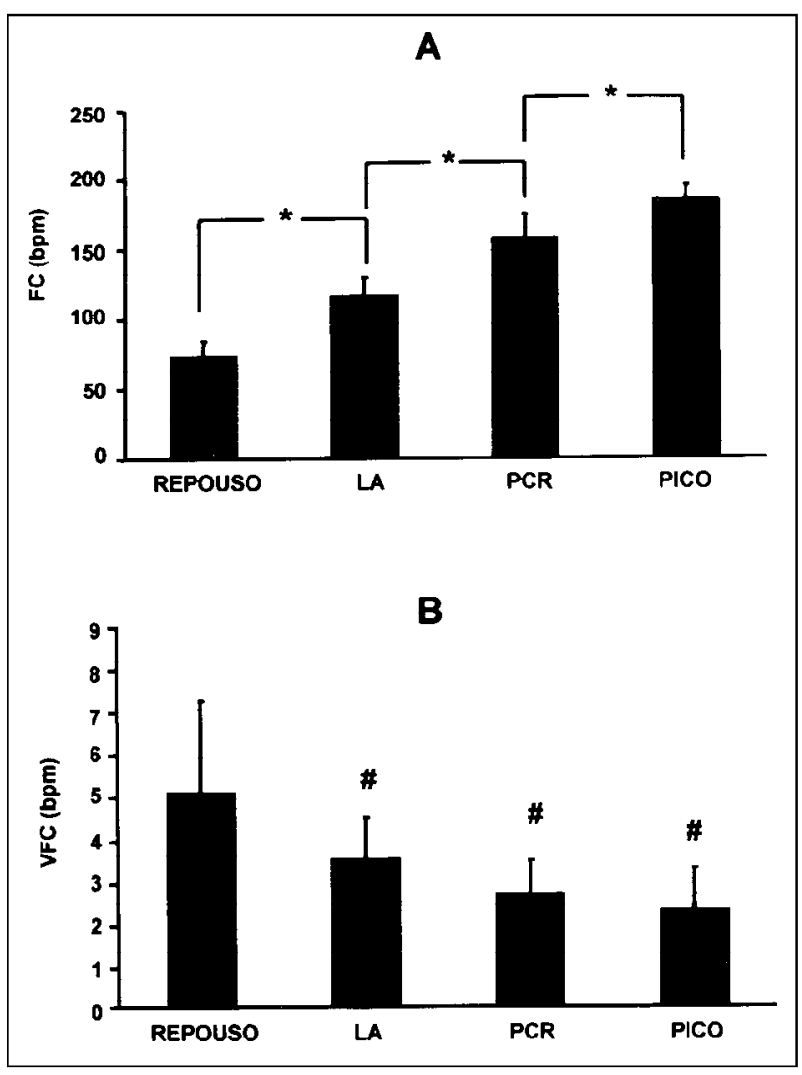

Fig. 1 - Comportamento da frequiência cardíaca (FC) em relação ao impacto metabólico do exercício físico progressivo máximo (painel A). Comportamento da variabilidade da frequiência cardíaca (VFC) em relação ao impacto metabólico do exercício físico progressivo máximo (painel B). LA- limiar anaeróbio; PCR- ponto de compensação respiratória; PICO- pico do exercício;* - diferença significante entre estágios $(\mathrm{P}<0,05)$ e, \# - diferença significante em relação ao repouso $(\mathrm{P}<0,05)$. máxima (FCmax). OPCR ocorreu em $75 \pm 9 \%$ do $\mathrm{VO}_{2}$ picoeem $89 \pm 8 \%$ daFCmax.

Em relação à Wmax atingida, observou-se que, na potência em que ocorreu oLA, os indivíduos se encontravam no intervalo de 45 a $60 \%$ da Wmax (47 $49 \%$ Wmax), sendo que a potência média no LA foi de $94 \pm 26 \mathrm{~W}$. No PCR, os indivíduos encontravam-se no intervalo de 70 a $85 \%$ da Wmax $(81 \pm 12 \%$ Wmax) e a potência média no PCR foi de $162 \pm 38 \mathrm{~W}$.

A FC aumentou significantemente $(\mathrm{P}<0,05)$ do repouso ao LA (73 \pm 11 vs $115 \pm 14 \mathrm{bpm})$, do LA para o PCR $(115 \pm 14$ vs $156 \pm 18 \mathrm{bpm}$ ) e do PCR ao pico de exercício (156 $\pm 18 v s$ $183 \pm 11$ bpm, fig. 1A).

A VFC diminuiu significantemente $(\mathrm{P}<0,05)$ do repouso para o LA $(5,0 \pm 2,2$ vs 3,4 $\pm 1,0 \mathrm{bpm}) \mathrm{e}$, a partir desta intensidade, manteve-se inferior ao repouso, sem queda adicional significante, até o final do exercício (fig. 1B).

AFC aumentou de forma significativa $(\mathrm{P}<0,05)$, a cada intervalo, desde o intervalo de $10 \%$ do $\mathrm{VO}_{2}$ pico até o pico do exercício, exceto do intervalo de $20 \%$ para o intervalo de $30 \%$ do $\mathrm{VO}_{2}$ pico (fig. 2A).

A VFC diminuiu significantemente $(\mathrm{P}<0,05)$ em relação ao repouso - que correspondeu a $10 \%$ do $\mathrm{VO}_{2}$ pico - a partir da intensidade de $60 \%$ do $\mathrm{VO}_{2}$ pico. Nas intensidades superiores, a VFC manteve-se diminuída em relação ao repouso até o final do exercício, porém sem queda adicional (fig. 2B).

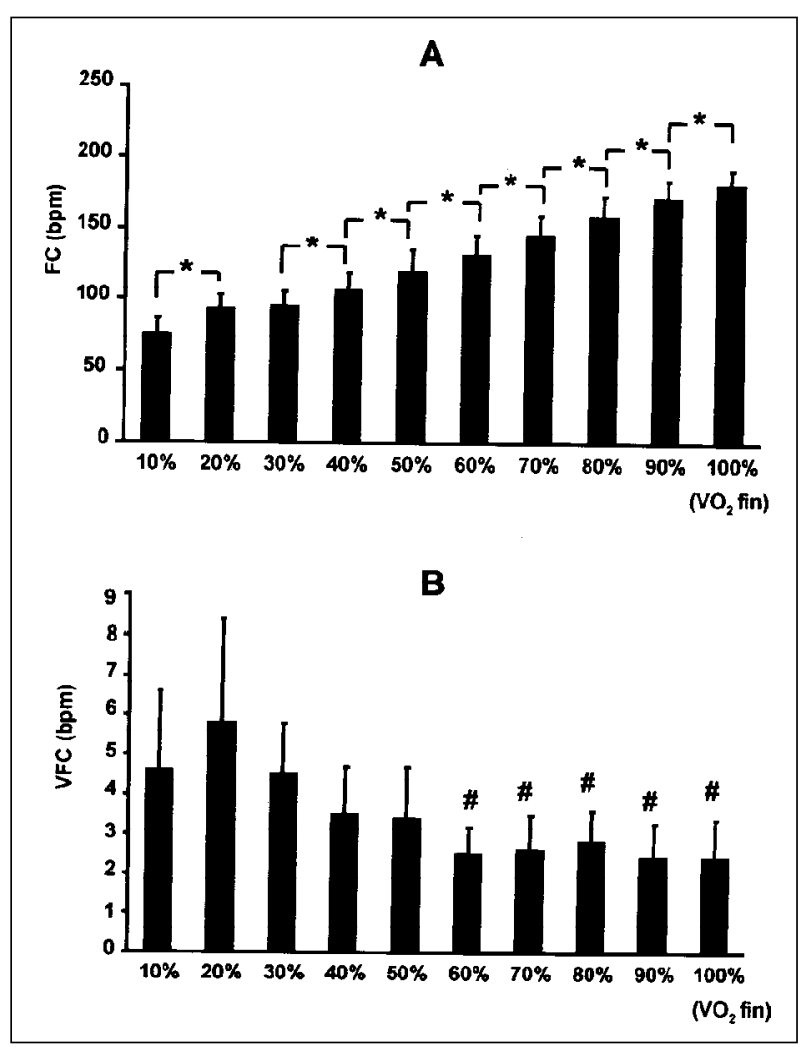

Fig. 2 - Comportamento da freqüência cardíaca (FC) em relação ao aumento relativo no consumo de oxigênio ( $\% \mathrm{VO}_{2}$ fin) durante o exercício físico progressivo máximo (painel A). Comportamento da variabilidade da freqüência cardíaca (VFC) em relação ao aumento relativo no consumo de oxigênio $\left(\% \mathrm{VO}_{2}\right.$ fin) durante o exercício físico progressivo máximo (painel B). $\mathrm{VO}_{2}$ fin- consumo máximo de oxigênio calculado por regressão linear; *- diferença significante entre estágios $(\mathrm{P}<0,05)$ e, \# - diferença significante em relação ao repouso $(\mathrm{P}<0,05)$. 
Na análise em relação ao aumento relativo de potência, a FC apresentou aumento progressivo e significante $(\mathrm{P}<0,05)$ entre os intervalos: repouso e 20 a $35 \%$ da Wmax (75 \pm 12 vs $101 \pm 12 \mathrm{bpm}) ; 20$ a $35 \%$ e 45 a $60 \%$ da $W \max$ (101 \pm 12 vs $128 \pm 15 \mathrm{bpm}) ; 45 \mathrm{a} 60 \mathrm{e} 70 \mathrm{a} 85 \%$ daWmax $(128 \pm 15$ vs $156 \pm 10 \mathrm{bpm}) ; \mathrm{e} 70$ a $85 \%$ da Wmax e a intensidade máxima de potência (156 \pm 10 vs $181 \pm 11 \mathrm{bpm})$ (fig. 3A).

A VFC diminuiu significantemente $(\mathrm{P}<0,05)$, em relação ao repouso, a partir do intervalo de $45 \%$ a $60 \%$ da Wmax $(4,6 \pm 2,0$ vs $2,9 \pm 0,8 \mathrm{bpm})$, mantendo-se diminuída até o final do exercício, sem queda adicional (fig. 3B).

\section{Discussão}

As principais verificações do presente estudo são: 1) a constatação de que apesar da VFC diminuir desde os primeiros instantes do exercício, essa redução somente atinge níveis expressivos a partir de $60 \%$ do $\mathrm{VO}_{2}$ pico, de $45-60 \%$ da Wmax e do LA; 2) a partir desses pontos do exercício a VFC tende a se estabilizar.

O aumento concomitante da FC e do consumo de oxigênio, observados no presente estudo, confirmam os resultados encontrados na literatura ${ }^{14,15,26}$. Essa relação entre a FC e o consumo de oxigênio demonstram que o comportamento da FC durante o exercício depende, em grande parte, da demanda metabólica na musculatura ativa ${ }^{27}$.
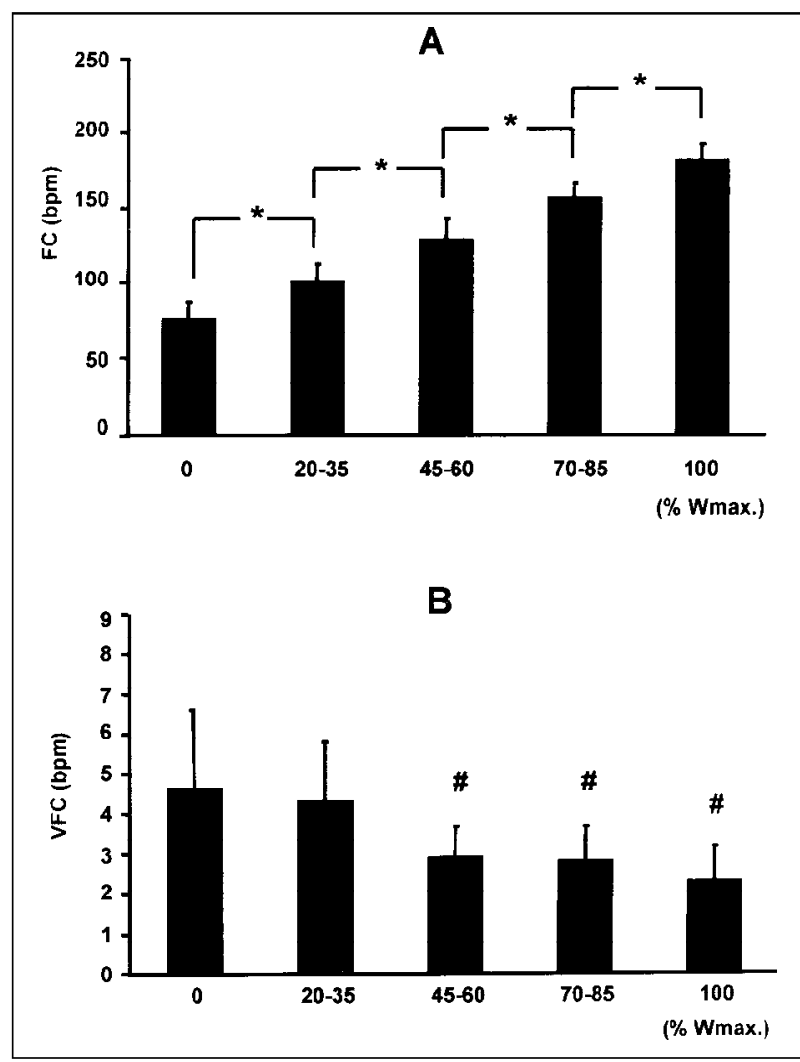

Fig. 3 - Comportamento da freqüência cardíaca (FC) em relação ao aumento da potência relativa (\%Wmax) durante o exercício físico progressivo máximo (painel A). Comportamento da variabilidade da frequiência cardíaca (VFC) em relação ao aumento da potência relativa (\%Wmax) durante o exercício físico progressivo máximo (painel B). WWatts; * - diferença significante entre estágios $(\mathrm{P}<0,05)$ e, \# = diferença significante em relação ao repouso $(\mathrm{P}<0,05)$.
Oexercício físico, além de alterações metabólicas, provoca importantes alterações autonômicas que influenciam o funcionamento cardiovascular ${ }^{12,13,26}$. Gallo e col $^{12}$, estudando a regulação autonômica da $\mathrm{FC}$ em diferentes estágios do exercício, observaram que a taquicardia inicial no exercício depende, principalmente, de uma retirada vagal, enquanto $o$ adicional de incremento na FC numa dada carga absoluta depende de um incremento do sistema nervoso simpático. Mais recentemente, verificamos, em animais de experimentação, que a atividade vagal diminuía progressivamente durante o exercício com cargas absolutas crescentes, enquanto a atividade nervosa simpática aumentava concomitantemente ${ }^{24}$. Esses resultados sugerem que a taquicardia do exercício é vago-dependente nos primeiros instantes e simpático-dependente nos períodos mais tardios.

A retirada vagal que controla o coração, no início do exercício, tem sido explicada pela irradiação cortical sobre a região bulbar ${ }^{12}$, enquanto a intensificação simpática no coração tem sido explicada pela ação de catecolaminas circulantes ${ }^{14} \mathrm{e}$ pela ação metabolorreflexa aferente iniciada na musculatura esquelética ativa ${ }^{13,28}$. Todos esses mecanismos agem em sintonia para atender a demanda metabólica na musculatura esquelética ativa.

No presente estudo, a análise simultânea da FC e da VFC, durante o exercício físico progressivo, demonstrou existir uma relação inversa, mas não linear, entre elas. A FC aumentou progressivamente até o pico do exercício, enquanto a VFC diminuiu progressivamente até o LA e, a partir desse ponto, manteve-se inalterada até o pico do exercício. Esses resultados sugerem que a diminuição da VFC é dependente, em grande parte, da retirada vagal. De fato, Yamamoto e col ${ }^{16,29}$, estudando a VFC durante o exercício físico, observaram diminuição do controle nervoso parassimpático no coração até o LA e aumento do controle nervoso simpático em intensidades acima do LA. Da mesma forma, Breuer e col ${ }^{18}$ observaram, através da análise dos componentes de baixa e alta freqüência da análise espectral e da relação entre eles que, no início do exercício, o aumento da FC é vago-dependente, enquanto nas fases mais avançadas do exercício ele é simpático-dependente. Alternativamente, é possível supor que a intensificação nervosa simpática no coração, após o LA, provoca uma taquicardia tão intensa que praticamente elimina a queda progressiva da VFC. No presente estudo, no LA e no PCR a FC era de $115 \pm 14$ e $156 \pm 18 \mathrm{bpm}$, respectivamente, o que demonstra um aumento expressivo da FC, com o aumento da intensidade do exercício. Em estudo recente do nosso laboratório ${ }^{30}$, observamos que já no LA as velocidades de enchimento e de esvaziamento ventricular esquerdo aumentavam significativamente demonstrando que, a partir de um certo ponto do exercício, os períodos destinados às fases diastólica e sistólica são drasticamente reduzidos. Comportamento semelhante pode perfeitamente ter ocorrido entre os ciclos cardíacos, o que levaria a uma diminuição na VFC.

Os resultados deste estudo têm uma implicação clínica: eles sugerem que a prática de exercício físico em intensi- 
dades até o LA parece proporcionar ao praticante maior segurança em relação a possíveis riscos cardiovasculares, uma vez que, nestas intensidades, ainda está presente a ação protetora vagal.

Embora outros estudos já tenham se dedicado a investigar a VFC durante o exercício, alguns aspectos metodológicos podem ter limitado suas interpretações a respeito do assunto em questão. Bernardi e $\mathrm{col}^{23}$ limitaram-se a estudar a VFC de indivíduos sedentários, atletas e transplantados em potências absolutas mas não em potências relativas. É possível que a estratificação de capacidade física inter-indivíduos fizesse com que a VFC fosse estudada em fases relativas distintas. Isto, obviamente, poderia limitar a interpretação dos resultados encontrados naquele estudo ${ }^{23}$. Outros autores ${ }^{16,17,20,21}$ avaliaram a VFC durante protocolos com incrementos de potência em rampa, nos quais os intervalos entre as potências não eram suficientes para que se atingisse uma condição de equilíbrio da FC. No presente estudo, embora a amostragem tenha sido limitada a indivíduos jovens sedentários, foi utilizado um protocolo escalonado e analisou-se a VFC em relação à potência relativa e não em relação à potência absoluta, para que uma possível estratificação de níveis de capacidade física individual não interferisse na interpretação dos nossos resultados ${ }^{31}$. Além disso, a presente abordagem metodológica possibilitou analisar a VFC em di- ferentes fases metabólicas do exercício físico progressivo.

Limitações - O protocolo escalonado, empregado na presente investigação, com incremento de carga a cada $3 \mathrm{~min}$, permitiu que a $\mathrm{FC}$ atingisse um estado de equilíbrio no $3^{\circ}$ minuto de cada estágio do exercício. Isso ocorreu nos primeiros estágios (até o LA), evitando que a VFC fosse influenciada pelo aumento da $\mathrm{FC}$ frente à elevação da potência mecânica da bicicleta. Entretanto, após o LA, essa condição de equilíbrio não é mantida, o que é inerente às altas intensidades de exercício. Dessa forma, é possível que em intensidades de exercício após o LA, a nossa interpretação a respeito da VFC seja limitada.

A hiperventilação e a vasodilatação periférica ${ }^{19}$, além de alterações intrínsecas ao músculo cardíaco ${ }^{23}$, podem também modular a FC durante o exercício. Assim, a VFC pode não ser uma maneira absoluta de se estudar o controle neural do coração durante o exercício progressivo máximo.

Concluindo, durante o exercício progressivo, a FC aumenta concomitantemente ao aumento no consumo de oxigênio e ao aumento de potência relativa. A VFC diminui progressivamente até o LA, $60 \%$ do $\mathrm{VO}_{2}$ pico e $45-60 \%$ da Wmax, mas se mantém inalterada a partir dessas intensidades de exercício. Estes resultados demonstram que a queda da VFC ocorre durante a fase do exercício em que predomina o metabolismo aeróbio como fonte de energia.

\section{Referências}

1. Van-Ravenswaaij-Arts CMA, Kollée LAA, Hopman CW, Stoelinga GBA, Geijn, HP - Heart rate variability. Ann Int Med 1993; 118: 436-47.

2. Bootsma M, Swenne CA, Van-Bolhuis HH, Chang PC, Cats VM, Bruschke AVG. - Heart rate and heart rate variability as indexes of sympathovagal balance. Am J Physiol 1994; 266: H1565-71.

3. Stein PK, Bosner MS, Kleiger RE, Conger BM - Heart rate variability: A measure of cardiac autonomic tone. Am Heart J 1994; 127: 1376-81.

4. Kleiger RE, Stein PK, Bosner MS, Rottman JN - Time-domain measurements of heart rate variability. In: Malik M, Camm AJ, eds - Heart rate variability. New York: Futura Publishing, 1995: 40.

5. Kleiger RE, Miller JP, Bigger Jr JT, Mois AJ - Decreased heart rate variability and its association with increased mortality after acute myocardial infarction. Multicenter post-infarction research group. Am J Cardiol 1987; 59: 256-62.

6. Bigger Jr JT, Fleiss JL, Steinman RC, Rolnitzky LM, Kleiger RE, Rottman JN Frequency domain measures of heart period variability and mortality after myocardial infarction. Circulation 1992; 85: 164-71.

7. Binder T, Frey B, Porenta G et al - Prognostic value of heart rate variability in patients awaiting cardiac transplantation. PACE 1992; 15: 2215-20.

8. Vaishnav S, Stevenson R, Marchant B, Lagi K, Ranjadayalan K, Timmis AD Relation between heart rate variability early after acute myocardial infarction and long-term mortality. Am J Cardiol 1994; 73: 653-7.

9. Grossman P, Karamaker J, Wieling W - Prediction of tonic parasympathetic cardiac control using respiratory sinus arrhythmia: The need for respiratory control. Psychophysiology 1991; 11: 277-90.

10. Ahmed MW, Kadish AH, Parker MA, Goldberger JJ - Effect of physiologic and pharmacologic adrenergic stimulation on heart rate variability. J Am Coll Cardiol 1994; 24 : 1082-90.

11. Mukai S, Hayana $\mathbf{J}$ - Heart rate and blood pressure variabilities during graded head-up tilt. J Appl Physiol 1995; 78: 212-6.

12. Gallo Jr L, Maciel BC, Marin Neto JA, Martins LEB - Sympathetic and parasympathetic changes in heart rate control during dynamic exercise induced by endurance training in man. Brazilian J Med Biol Res 1989; 22: 631-43.

13. Mitchell JH - Neural control of the circulation during exercise. Med Sci Sports Exer 1990; 22: 141-54.

14. Rowell LB - Human circulation: regulation during physical stress. New York: Oxford University Press, 1986: 213-17.
15. McArdle WD, Katch FI, Katch VL - Exercise Physiology. Energy, nutrition and human performance. $3^{\text {rd }}$ ed. Philadelphia: Lea \&Febiger, 1991: 313.

16. Yamamoto Y, Hughson RL, Peterson JC - Autonomic control of heart rate during exercise studied by heart rate variability spectral analysis. J Appl Physiol 1991; 71: 1136-42

17. Nakamura Y, Yamamoto Y, Muraoka I - Autonomic control of heart rate during physical exercise and fractal dimension of heart rate variability. J Appl Physiol 1993; 74: 875-81.

18. Breuer H-W M, Skyschally A, Schulz R, Martin C, Wehr M, Heusch G-Heart rate variability and circulating catecholamine concentrations during steady state exercise in healthy volunteers. Br Heart J 1993; 70: 144-9.

19. Rimoldi O, Furlan R, Pagani MR et al - Analysis of neural mechanisms accompanying different intensities of dynamic exercise. Chest 1992; 101(suppl): 226-30.

20. Shin K, Minamitami H, Onishi S, Yamazaki H, Lee M - The power spectral analyses of heart rate variability in athletes during dynamic exercise - Part I. Clin Cardiol 1995; 18: 583-6.

21. Shin K, Minamitami H, Onishi S, Yamazaki H, Lee M - The power spectral analyses of heart rate variability in athletes during dynamic exercise - Part II. Clin Cardiol 1995; 18: 664-8.

22. Arai $Y$, Saul JP, Albrecht $P$ et al - Modulation of cardiac autonomic activity during and immediately after exercise. Am J Physiol 1989; 256: H132-41.

23. Bernardi L, Salvucci F, Suardi R et al - Evidence for an intrinsic mechanism regulating heart rate variability in the transplanted and the intact heart during submaximal dynamic exercise? Cardiovasc Res 1990; 24: 969-81.

24. Coumel P, Maison-Blanche P, Catuli D - Heart rate and heart rate variability. In: Malik M, Camm AJ, eds - Heart Rate Variability. New York: Futura Publishing, 1995: 207

25. Skinner JS, McLellan TH - The transition from aerobic to anaerobic metabolism. Res Q Exerc Sport 1980; 51: 234-48.

26. Negrão CE, Moreira ED, Brum PC, Denadai ML, Krieger EM - Vagal and sympathetic control of heart rate during exercise by sedentary and exercisetrained rats. Brazilian J Med Biol Res 1992; 25: 1045-52.

27. Lewis SF, Taylor WF, Graham RM, Pettinger WA, Schutte JE, Blomqvist CG Cardiovascular responses to exercise as functions of absolute and relative work load. J Appl Physiol 1983; 54: 1314-23. 
28. Middlekauff HR, Nguyen AH, Negrão CE et al - Impact of acute mental stress on sympathetic nerve activity and regional blood flow in advanced heart failure: implications for "triggering" adverse cardiac events. Circulation 1997; 96: 1835-42.

29. Yamamoto Y, Hughson RL, Nakamura Y - Autonomic nervous system responses to exercise in relation to ventilatory threshold. Chest 1992; 101(suppl): 206-10
30. Brandão UM, Wajngarten M, Rondon E, Giorgi MC, Hironaka T, Negrão CE-Left ventricular function during dynamic exercise in untrained and moderately trained subjects. J Appl Physiol 1993; 75: 1989-95.

31. Rezende LO, Santaella DF, Forjaz CLM, Negrão CE - Comportamento da variabilidade da frequência cardíaca durante o exercício progressivo. Anais do $3^{\circ}$ Congresso Nacional do Departamento de Ergometria e Reabilitação Cardiovascular da Sociedade Brasileira de Cardiologia 1996; 23. 